\title{
Metaheurísticas Aplicadas a la Ubicación y Dimensionamiento Óptimo de Generación Distribuida en Sistemas de Distribución de Energía Eléctrica
}

\author{
Jesús M. López-Lezama, Fernando Villada y Nicolás Muñoz \\ Universidad de Antioquia, Departamento de Ingeniería Eléctrica, Calle 70 No 52-21, Medellín - Colombia \\ (e-mail: jmaria.lopez@udea.edu.co; fernando.villada@udea.edu.co; nicolas.munoz@udea.edu.co).
}

Recibido Nov. 6, 2014; Aceptado Ene. 13, 2015; Versión final Mar. 28, 2015, Publicado Ago. 2015

\begin{abstract}
Resumen
En este artículo se presenta una evaluación de la ubicación y dimensionamiento óptimo de generación distribuida en sistemas de distribución de energía eléctrica. Para tal evaluación se han considerado las siguientes técnicas de búsqueda: i) recocido simulado, ii) búsqueda de entorno variable iii) algoritmo genético y iv) algoritmo genético híbrido. En el problema de ubicación y dimensionamiento óptimo se han considerado dos objetivos: la minimización de pérdidas activas y el mejoramiento del perfil de tensiones. Para comparar la efectividad de los métodos implementados se utiliza un sistema de distribución de prueba de 34 barras. La correcta ubicación y dimensionamiento de la generación distribuida permitió mejorar sustancialmente el perfil de tensión de la red y reducir en un $80 \%$ las pérdidas para el sistema de prueba.
\end{abstract}

Palabras clave: generación distribuida, algoritmos genéticos, entorno variable, recocido simulado

\section{Metaheuristics Applied to the Optimal Location and Sizing of Distributed Generation in Power Distribution Systems}

\begin{abstract}
This paper presents an assessment of optimal location and sizing of distributed generation in distribution systems. For such an assessment the following search techniques were implemented: i) simulated annealing, ii) variable neighborhood search, iii) genetic algorithm and iv) hybrid genetic algorithm. Two objectives have been considered in the optimal location and sizing problem: the minimization of electric power losses and the improvement of voltage profile. To compare the effectiveness of the methodologies a 34 bus distribution test system is used. The correct location and sizing of the distributed generation allowed substantially improving the voltage profile of the network and reducing in $80 \%$ the power losses in the test system.
\end{abstract}

Keywords: distributed generation, genetic algorithms, variable neighborhood, simulated annealing 


\section{INTRODUCCIÓN}

En la última década ha habido profundos cambios en el sector eléctrico. Dentro de estos cambios se destaca la creciente participación de Generación Distribuida (GD) en las redes de distribución. Este fenómeno ha sido motivado por diversos factores que incluyen: nuevos avances tecnológicos en la producción de electricidad a pequeña escala, preferencia por el uso de recursos renovables, dificultades en la ampliación de redes y un creciente interés por incorporar la demanda como agente activo (mediante la autoproducción) en los mercados de electricidad (Jonghe et al., 2011). La GD (definida como la producción de electricidad cerca a los centros de consumo) puede contribuir a la reducción de pérdidas, al mejoramiento de perfil de tensiones, mejoramiento de la confiabilidad y aplazamiento de inversiones en redes de transmisión y distribución (Hung et al., 2010). Sin embargo, como se anota en (Hung y Mithualananthan, 2013), el aprovechamiento de los beneficios de la GD depende en gran medida de su ubicación, dimensionamiento y características de la red. Es por esto que en la última década se han explorado diversas metodologías para su apropiada ubicación y dimensionamiento.

En (Georgilakis y Hatziargyriou, 2013) se presenta una revisión bibliográfica de las técnicas utilizadas para la ubicación y dimensionamiento óptimo de GD en redes de distribución. Los autores clasifican las técnicas en métodos analíticos, metaheurísticas, y programación matemática. Cabe anotar que para el problema bajo estudio las técnicas metaheurísticas presentan ventajas significativas frente a la programación matemática clásica en razón de la naturaleza no lineal y no convexa de la ubicación y dimensionamiento de la GD. Por otro lado, la debilidad principal de las metaheurísticas radica en que no garantizan la obtención de un óptimo global. Sin embargo, permiten encontrar una solución o un conjunto soluciones de alta calidad (Leopoldo y Monzón, 2010). Otra ventaja de las técnicas metaheurísticas radica es que permiten el uso de modelos más detallados de operación de la red como el modelo AC. En contraste, para aplicar técnicas de programación matemática al problema de ubicación o dimensionamiento óptimo de GD es necesario utilizar linealizaciones o aproximaciones a las ecuaciones de balance de potencia y flujos de carga como ilustrado en (Lopez-Lezama et al., 2011).

Las técnicas de optimización metaheurística han sido ampliamente utilizadas en la ubicación y dimensionamiento de GD. Dentro de estas se destacan los Algoritmos Genéticos, la Búsqueda Tabú y colonias de partículas, entre otras. En (Aly et al., 2010) se presenta un modelo de optimización multiobjetivo para determinar la ubicación y dimensionamiento óptimo de GD mediante la técnica de enfriamiento simulado. Los objetivos modelados son las pérdidas de potencia, el número de generadores, el perfil de tensión y la potencia inyectada por la GD. En este sentido, se busca encontrar una solución que permita mejorar el perfil de tensiones y reducir las pérdidas con el mínimo de unidades de GD en el sistema. En (Tan et al., 2012) los autores presentan una metaheurística poblacional basada en el comportamiento reproductivo parasitario de algunas especies de cucos (Cuckoo Search Algoritm) para la ubicación y dimensionamiento óptimo de GD. La ventaja de este algoritmo consiste en que requiere de pocos parámetros para calibrar. El objetivo del estudio consiste en la reducción de pérdidas de potencia activa.

En (Akorede et al, 2011) se presenta un AG para ubicar y dimensionar de forma óptima varias unidades de GD con el objetivo de maximizar el margen de carga y los lucros de la empresa distribuidora. Los autores muestran la viabilidad económica de la GD referente al aplazamiento de inversiones en la red. En (Chen y Duan, 2014) y (Zhao et al., 2014) también se presentan otros estudios de ubicación y dimensionamiento de GD usando Algoritmos Genéticos. En (Maciel y Padilha-Feltrin, 2009) se presenta una Búsqueda Tabú multiobjetivo para evaluar el impacto de la GD considerando reducción de pérdidas, mejoramiento del perfil de tensiones y minimización del índice de corriente de corto circuito trifásico. En (Soroudi y Afrasiab, 2012) se presenta un método de búsqueda basado en colonias de partículas que busca optimizar simultáneamente tres objetivos: satisfacción de restricciones técnicas, reducción de costos y reducción de emisiones contaminantes. El método propuesto determina la ubicación, dimensionamiento y momento en el cual se deben hacer las inversiones en la red.

Los métodos híbridos combinan dos o más técnicas de búsqueda con el objetivo de aprovechar las potencialidades de estas y compensar sus deficiencias. Las técnicas híbridas más comunes combinan métodos poblacionales con métodos que intensifican algún tipo de búsqueda local o alternativamente, métodos heurísticos con programación matemática clásica. En (Gandomkar et al., 2005) se presenta una combinación de enfriamiento simulado y algoritmos genéticos para ubicación optima de GD en redes de distribución. El objetivo propuesto es la minimización de pérdidas. Los autores muestran que la combinación de AG con enfriamiento simulado resulta ser más efectivo el uso de solamente AG. En (Soroudi et al., 2011) se presenta un algoritmo híbrido genético-inmune para maximizar el beneficio de operadores de red y propietarios de generación distribuida en un mercado eléctrico desregulado. El modelo propuesto optimiza simultáneamente dos objetivos: los beneficios de la empresa distribuidora y del propietario de la GD. También considera la incertidumbre de la demanda y precios de energía. En (Tan et al., 2013) se presenta 
un algoritmo híbrido que combina colonia de partículas con búsqueda gravitacional para determinar la ubicación y dimensionamiento apropiados de la GD que minimice pérdidas, mejore perfiles de tensiones y reduzca emisiones contaminantes.

El objetivo de este artículo es contribuir a la discusión sobre la efectividad de los métodos heurísticos y metaheurísticos para la ubicación y dimensionamiento óptimo de GD. Para este fin, se han implementado y comparado cuatro técnicas diferentes i) Recocido Simulado ii) Búsqueda de Entorno Variable iii) Algoritmo Genético y iv) un método híbrido que combina la Búsqueda de Entorno Variable con un Algoritmo Genético. Para probar la eficiencia de estos métodos se han hecho diferentes pruebas en un sistema de distribución de 34 barras, ampliamente utilizado en la literatura técnica.

\section{FORMULACIÓN MATEMÁTICA}

La función objetivo del problema propuesto consiste en mejorar el perfil de tensiones y reducir las pérdidas del sistema. Para tal fin se tomaron los índices definidos en (Chiradeja et al., 2004) que se describen a continuación. El índice de perfil de tensiones, denotado como IPT, está definido por la ecuación (1). Este índice no solamente tiene en cuenta la tensión en las barras sino también la carga de las mismas como factor de peso. De esta forma resulta más importante mantener tensiones apropiadas en las barras con mayor demanda.

$I P T=\sum_{i=1}^{N} V_{i} L_{i}$

Variables: $V_{i}$ : Tensión en la barra i (p.u); $L_{i}$ : Carga en la barra i (p.u); y $N$ : Número total de barras.

El índice de mejoramiento del perfil de tensiones, denotado como IMPT está dado por la ecuación (2).

$I M P T=\frac{I P T_{w D G}-I P T_{w o D G}}{I P T_{w o D G}} \times 100$

Variables: $I P T_{w D G}$ es el índice de perfil de tensiones del sistema con GD (p.u.) y $P T_{w o D G}$ es el índice de perfil de tensiones del sistema sin GD (p.u.). Note que el IMPT denota el mejoramiento en porcentaje del IPT con $y \sin G D$.

El segundo objetivo es la reducción de pérdidas activas, para ello se comparan las pérdidas en el sistema con y sin GD dadas por las expresiones (3) y (4), respectivamente.

$I P L_{w D G}=\sum_{K=1}^{N} I^{2}{ }_{K, W D G} R_{k} D_{k}$

$I P L_{w o D G}=\sum_{K=1}^{N} I^{2}{ }_{K, W o D G} R_{k} D_{k}$

Variables:

$I P L_{w D G}$ : Índice de pérdidas en las líneas considerando GD.

$R_{k}$ : Resistencia en la línea $\mathrm{k}(\mathrm{p} . \mathrm{u} / \mathrm{km})$.

$D_{k}$ : Longitud de la línea $\mathrm{k}(\mathrm{km})$.

$I_{K, w D G}^{2}$ : Corriente de la línea k con GD (p.u).

$L L_{w o D G}$ : Índice de pérdidas en las líneas sin GD.

$I_{K, w o D G}^{2}$ : Corriente de la línea $\mathrm{k} \sin \mathrm{GD}(\mathrm{p} . u)$.

El índice de reducción de pérdidas en las líneas IRPL está dado por la ecuación (5):

$I R P L=\frac{I P L_{w o D G}-I P L_{w D G}}{I P L_{w o D G}} \times 100$

La función objetivo consiste en maximizar los índices de reducción de pérdidas y mejoramiento de perfil de tensiones. En este caso se deben utilizar factores de peso $\mathrm{w}_{1}$ y $\mathrm{w}_{2}$ adimensionales para cada uno de los índices con el propósito de valorar su importancia dentro del proceso de optimización. El problema de optimización a resolver es descrito por las ecuaciones (6) a (18). 
Maximizar $f(x)=w_{1} I R P L+w_{2} I M P T$

Sujeto a:

$0 \leq \mathrm{w}_{\mathrm{m}} \leq 1 ; \quad \mathrm{m}=1,2$

$\sum_{m=1}^{2} w_{m}=1$

$u_{i} P_{G i}-P_{D i}-V_{i} \sum_{k=1}^{n b}\left[V_{k}\left\{g_{i k} \cos \left(\theta_{i k}\right)+b_{i k} \sin \left(\theta_{i k}\right)\right\}\right]=0$

$u_{i} Q_{G i}-Q_{D i}-V_{i} \sum_{k=1}^{n b}\left[V_{j}\left\{g_{i k} \sin \left(\theta_{i k}\right)-b_{i k} \cos \left(\theta_{i k}\right)\right\}\right]=0$

$P_{i k}=V_{i}^{2} g_{i k}-V_{i} V_{k} g_{i k} \cos \left(\theta_{i k}\right)-V_{i} V_{k} b_{i k} \sin \left(\theta_{i k}\right)$

$Q_{i k}=V_{i}^{2} b_{i k}-V_{i} V_{k} b_{i k} \cos \left(\theta_{i k}\right)-V_{i} V_{k} b_{i k} \sin \left(\theta_{i k}\right)$

$S_{i k}^{2}=P_{i k}^{2}+Q_{i k}^{2}$

$P_{G j}^{\min } \leq P_{G j} \leq P_{G j}^{\max }$

$V_{i}^{\min } \leq V_{i} \leq V_{i}^{\max }$

$S_{i k} \leq S_{i k}^{\max }$

$N_{G D} \leq N_{G D}^{\max }$

$u_{i} \in\{0,1\}$

Donde $P_{G i}$ y $Q_{G i}$ son las potencias activa y reactiva respectivamente, entregadas por una unidad de GD si está ubicada en la barra $i$. Note que no todas las barras tendrán GD y para cada unidad de GD habrá que asignar una variable binaria (denominada $u_{i}$ ). Por simplicidad, no se ha considerado que la GD inyecte o tome potencia reactiva de la red, por lo que $Q_{G i}=0$. $P_{D i}$ y $Q_{D i}$ corresponden a las demandas de potencia activa y reactiva en la barra $i$, respectivamente. $n b$ es el número de barras, $\theta_{i k}$ es la apertura angular entre las barras $i$ y $k$; $g_{i k}$ y $b_{i k}$ son las partes real e imaginaria, respectivamente, de la matriz de admitancia nodal. Las restricciones (9) y (10) representan las ecuaciones de balance de potencia activa y reactiva, respectivamente. Las restricciones (11), (12) y (13) representan las ecuaciones de flujo de potencia activa, reactiva y aparente, respectivamente. Las restricciones (14), (15) y (16), consideran los límites de potencia inyectada por la GD, los límites de tensión en la red y los límites de flujo de carga, respectivamente. La restricción (17) indica el número máximo de unidades de GD a tener en cuenta y la restricción (18) indica la naturaleza binaria de las variables $u_{i}$ ( 1 si hay GD y 0 si no hay GD).

El modelo descrito por las ecuaciones (6)-(18) corresponde a un problema de programación no lineal entero mixto, altamente dimensional y no convexo que presenta múltiples óptimos locales, lo cual justifica para su solución, el uso de los métodos de búsqueda ilustrados en este artículo. 


\section{METODOLOGIA}

Para abordar el problema de ubicación y dimensionamiento óptimo de GD descrito en la sección anterior se utilizaron cuatro técnicas de optimización combinatorial: Recocido Simulado, Búsqueda de Entorno Variable Descendente, Algoritmo Genético y Algoritmo Genético Híbrido. A continuación se presenta una breve descripción de las técnicas de solución adoptadas en este artículo.

\section{Recocido Simulado (RS)}

EI RS o enfriamiento simulado se basa en la emulación del recocido del acero y cerámicas, una técnica que consiste en calentar y luego enfriar lentamente el material para variar sus propiedades físicas. Este procedimiento fue introducido en 1983 por Kirkpatric y colaboradores (Kirkpatrick et al., 1983). En cada iteración del RS se evalúan algunos vecinos del estado actual y probabilísticamente se decide entre efectuar la transición a un nuevo estado o quedarse en el estado actual.

Si la solución vecina mejora el valor de la función objetivo se acepta con probabilidad 1, en caso contrario se calcula la probabilidad para aceptarla mediante el criterio de Metropolis dado por la ecuación (19) donde el parámetro $c$, corresponde a la temperatura.

$\operatorname{Prob}\left(\operatorname{aceptar} x^{\prime}\right)=\left\{\begin{array}{cl}1 & , f\left(x^{\prime}\right)<f(x) \\ \exp \left(-\frac{f\left(x^{\prime}\right)-f(x)}{c}\right), & f\left(x^{\prime}\right) \geq f(x)\end{array}\right.$

EI RS permite evaluar soluciones poco atractivas en los primeros pasos, luego a medida que el parámetro de temperatura se reduce, la búsqueda se vuelve más selectiva, disminuyendo los empeoramientos de la función objetivo. La mejor solución visitada será la solución heurística dada por el algoritmo.

\section{Búsqueda de Entorno Variable Descendente (BEVD)}

La Búsqueda de Entorno Variable (BEV) es una metaheurística que se fundamenta el cambio de vecindad (también conocida como estructura de entorno) en una búsqueda local (Hansen et al., 2003). La BEV tiene diferentes variaciones, recibiendo el nombre de BEV descendente, reducida, básica o general.

En este artículo se implementó una extensión de BEV llamada Búsqueda de Entorno Variable Descendente (BEVD), en la cual se cambia la solución actual por la obtenida en una búsqueda local; siempre y cuando en esta se haya encontrado una mejor solución. El algoritmo para la BEVD se ilustra a continuación (Glover et al., 2003):

Inicialización: Seleccionar el conjunto de estructuras de entornos $\mathrm{Nk}, k=1, \ldots, k m a x$, que se usarán en el descenso. Encontrar una solución inicial $x$;

Iteraciones: Repetir, hasta que no se obtenga mejora, la siguiente secuencia: (1) Hacer $k \leftarrow 1$; y (2) Repetir, hasta que $k=k_{\max }$, los pasos: (a) Exploración del entorno: Encontrar la mejor solución $x^{\prime}$ del $k$-ésimo entorno de $x\left(x^{\prime} \in N_{k}(x)\right) ; y(b)$ Moverse o no: Si la solución obtenida $x^{\prime}$ es mejor que $\mathrm{x}$, hacer $x \leftarrow x^{\prime} \mathrm{y} k \leftarrow 1$; en otro caso, hacer $k \leftarrow k+1$.

En el estudio presentado en este artículo, los entornos o vecindades fueron definidos como el tamaño (aumentar o disminuir la capacidad de la GD) y la ubicación (mover la GD a un nodo vecino).

\section{Algoritmo Genético (AG)}

Los Algoritmos Genéticos hacen parte de las técnicas evolutivas que pueden ser usadas para resolver problemas de optimización. Este método está basado en el concepto de selección natural y la supervivencia del individuo más apto (Anaut et al., 2009). La rutina general de un AG consiste en generar una población inicial de forma aleatoria o pseudo-aleatoria. Cada individuo de la población es definido mediante una cadena de bits. En este caso, para evaluar la función objetivo se corre un flujo de potencia teniendo en cuenta la ubicación y dimensionamiento de la GD. Con los resultados del flujo se calculan los índices de pérdidas y niveles de tensiones. Para la selección se hacen torneos de un número dado de individuos. El número de torneos es igual al tamaño de la población. La recombinación se hace en un solo punto seleccionado de forma aleatoria. La mutación consiste en cambiar un bit (de cero a uno o viceversa) de forma aleatoria con una probabilidad de ocurrencia dada. Los individuos generados en el proceso de recombinación y mutación reemplazarán los individuos actuales si son mejores que sus predecesores. Se consideran dos criterios de parada: el número máximo de iteraciones o el número máximo de iteraciones sin mejora de la función objetivo. 


\section{Algoritmo Genético Híbrido (AGH)}

Los métodos híbridos buscan combinar las ventajas de dos o más metaheurísticas para obtener soluciones de alta calidad. Los híbridos más comunes combinan métodos poblacionales (Algoritmos Genéticos, algoritmos basados en colonias de hormigas, algoritmos basados en colonia de abejas) con métodos de búsqueda local (Recocido Simulado, Búsqueda de Entorno Variable), o métodos exactos (programación lineal, no lineal) con métodos heurísticos. En este trabajo se implementó un método poblacional (AG) combinado con un método de búsqueda local (BEVD). El diagrama de flujo del algoritmo implementado se ilustra en Fig.1. La estructura del AGH conserva esencialmente la misma del AG descrito en la sección anterior; sin embargo, después de la mutación y antes de reemplazar los individuos de la nueva generación se realiza una BEVD con el objetivo de encontrar individuos de mejor calidad en cada generación. Al igual que en el AG ya descrito, solo se incluyen en la nueva generación aquellos individuos que presenten mejora en la función objetivo.

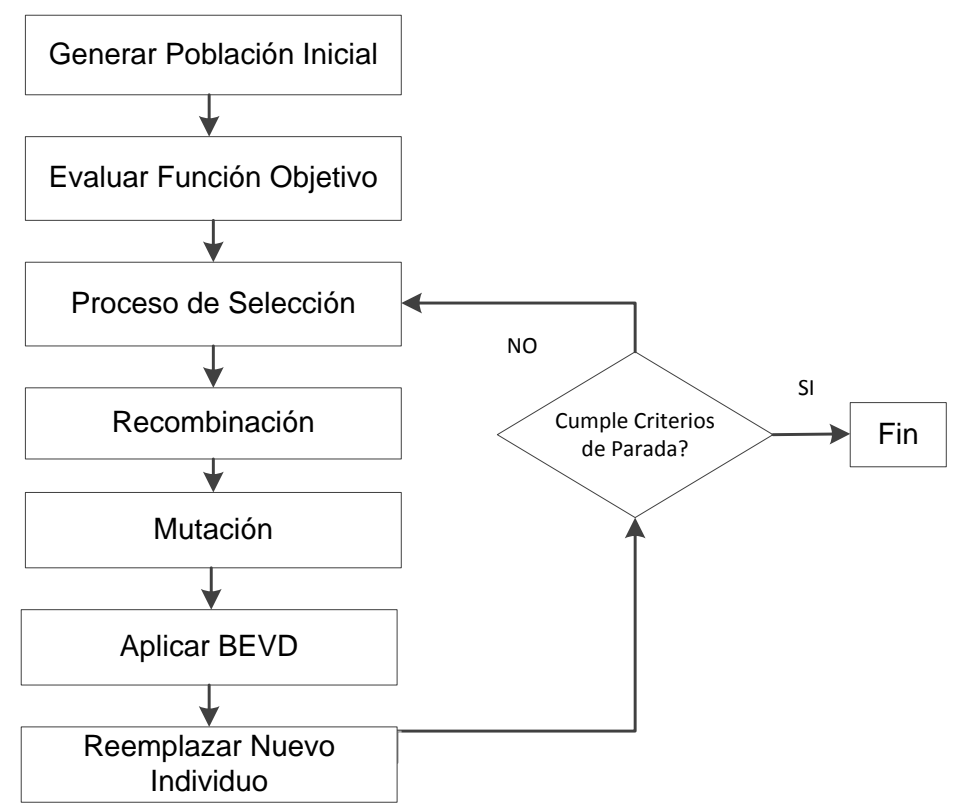

Fig.1: Diagrama del AGH

\section{RESULTADOS}

En la Fig. 2 se ilustra un sistema de distribución de 34 barras cuyos datos pueden ser consultados en (López-Lezama et al., 2011). La demanda máxima de la red es de 15,8MW con factor de potencia de 0,95 en atraso. Se considera que se pueden incluir hasta 4 unidades de GD, cada una con una potencia máxima de 2,0 MW. Las soluciones candidatas con más unidades de GD son penalizadas en la función objetivo con el fin de hacerlas menos atractivas. Note que según la formulación (ecuación (17)) es posible seleccionar otro número de unidades de GD como máximo.

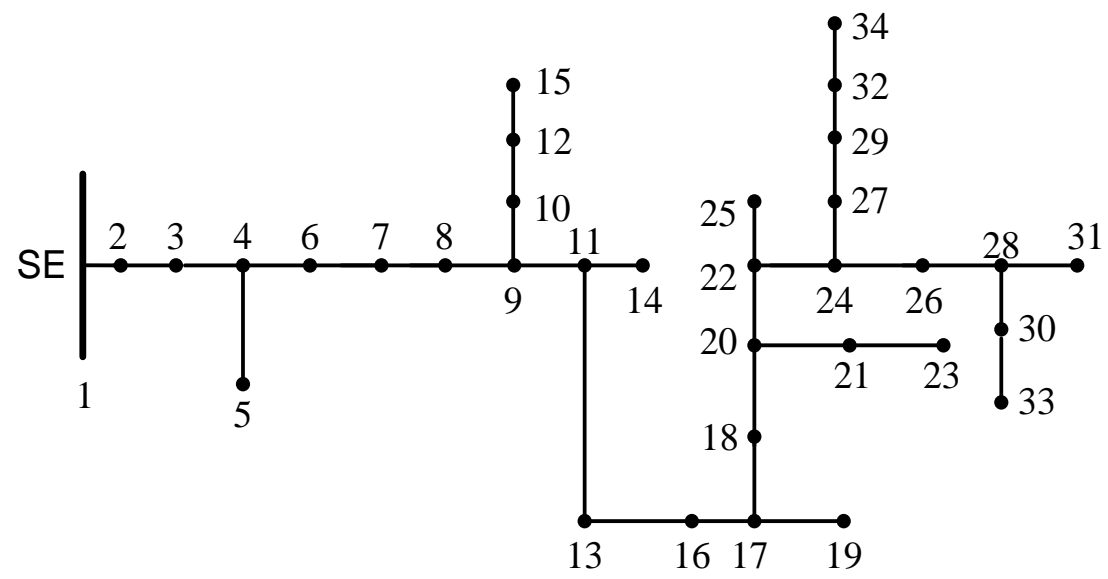

Fig.2: Sistema de distribución de 34 barras 
La distribución de la demanda en cada uno de los nodos de la red se ilustra en Fig. 3. Se puede observar que una parte significativa de la demanda se encuentra en las primeras barras. En todas las simulaciones se utilizó un computador Intel Core 2 Duo de $2,53 \mathrm{GHz}$ con 4 GB de RAM.

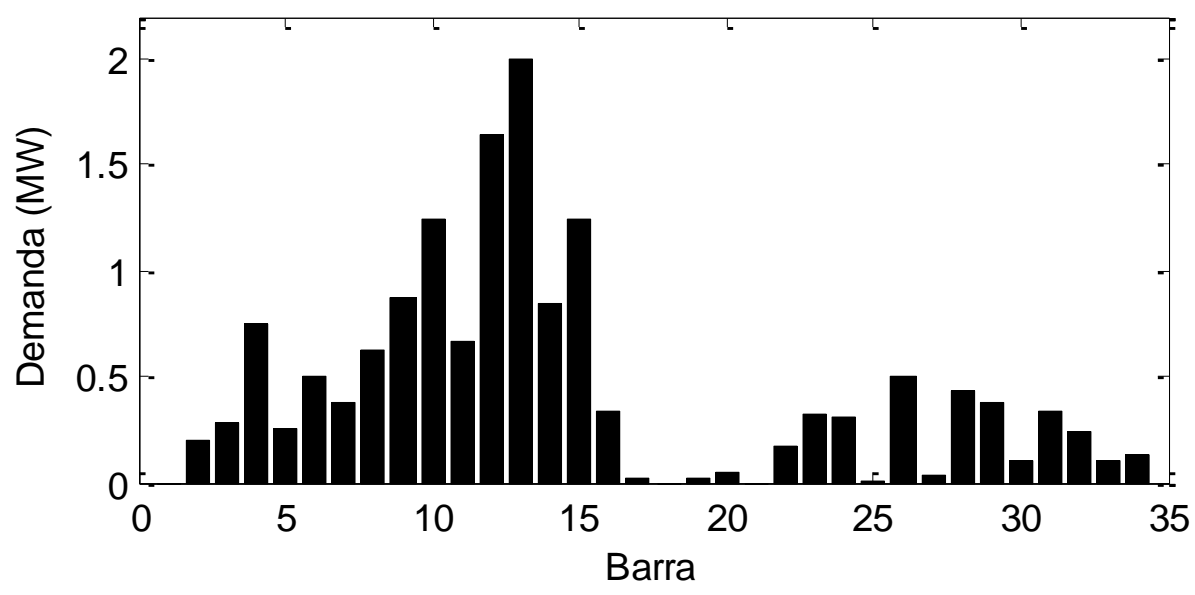

Fig.3: Distribución de la demanda en el sistema de 34 barras

\section{Codificación de las soluciones}

Para la codificación se utiliza una cadena de 4 bits binarios que representan cada generador. El primer bit representa el estado del generador (1: el generador existe, 0 el generador no existe); los 4 bits restantes representan el nivel de potencia del generador. Por ejemplo la cadena 1000 significa un generador de capacidad mínima $(0,25 \mathrm{MW})$, mientras la cadena 1111 representa un generador con capacidad máxima (2,0 MW). En la Tabla 1 se ilustran las equivalencias. En la Tabla 2 se ilustra la codificación de los candidatos de solución. Se utiliza una cadena binaria igual a $4^{*} \mathrm{nb}$, donde nb es el número de barras del sistema. Según la codificación adoptada, el candidato de solución ilustrado en la Tabla 2 no presenta GD en las barras 1,2, 4 y nb-1; mientras en la barra 3 y nb presenta generadores con capacidad de 1,0 y 2,0 MW, respectivamente.

Tabla 1: Codificación del tamaño de la GD

\begin{tabular}{|c|c|c|c|}
\hline Código & Tamaño (MW) & Código & Tamaño (MW) \\
\hline 1000 & 0,25 & 1100 & 1,25 \\
\hline 1001 & 0,50 & 1101 & 1,50 \\
\hline 1010 & 0,75 & 1110 & 1,75 \\
\hline 1011 & 1,00 & 1111 & 2,00 \\
\hline
\end{tabular}

Tabla 2: Codificación de los candidatos de solución

\begin{tabular}{|c|c|c|c|c|c|c|}
\hline Bus 1 & Bus 2 & Bus3 & Bus 4 & & Bus nb-1 & Bus nb \\
\hline 0100 & 0011 & 1011 & 0111 & $\ldots \ldots$. & 0011 & 1111 \\
\hline
\end{tabular}

Calibración de los Factores de Peso

Antes de iniciar el proceso de optimización se deben asignar factores de peso a los dos objetivos bajo estudio (IRPL e IMPT) con el fin de darles mayor o menor importancia en la búsqueda de soluciones. Para ello es necesario hacer un diagnóstico del caso base sin GD. Este diagnóstico se hace mediante un cálculo de flujo de carga con y sin GD. El flujo de carga se realiza mediante el software Matpower (Zimmerman et al., 2011) modelando la GD como barras donde se puede inyectar potencia activa. En Fig. 4 y Fig. 5 se ilustra el perfil de tensiones y las pérdidas en las líneas, respectivamente con y sin GD. Para ilustrar el caso con GD se suponen 4 unidades de GD, cada una de $1 \mathrm{MW}$ ubicadas en las barras 5, 10, 15 y 20. En Fig. 4 se puede observar que incluso ajustando la tensión en la subestación en 1.1 p.u, cuando no hay GD las tensiones en las últimas barras están por debajo de 0,9 p.u. presentando regulación de tensión (diferencia porcentual entre las tensiones máxima y mínima del sistema) superior al $20 \%$. Sin embargo, cuando se introduce GD al sistema las tensiones aumentan, especialmente en las barras más alejadas de la subestación, haciendo que la regulación de tensión en estas barras sea de $17,5 \%$. Por otro lado, las pérdidas totales del sistema sin GD ascienden a $959,5 \mathrm{~kW}$ y con GD son $555,8 \mathrm{~kW}$. Al calcular los índices 
IRPL e IMPT mediante las expresiones (2) y (5), respectivamente se obtiene que IRPL $=41,8848$ e IMPT= 1,5135. Estos índices son calculados para diferentes candidatos de solución (ubicación y dimensionamiento de GD) generados de forma aleatoria dando como resultado los datos consignados en la Tabla 3.

Se puede observar que IRPL es mucho mayor a IMPT en todas las simulaciones. Esto se da porque el porcentaje de reducción de pérdidas es más significativo que el mejoramiento en el perfil de tensiones. En promedio, IRPL es aproximadamente 20 veces mayor a IMPL como se puede apreciar en la cuarta columna de la Tabla 3. Si se optimizan los índices sin utilizar factores de peso se tendrían soluciones que privilegian la reducción de pérdidas sobre el mejoramiento de tensiones. En este caso se seleccionan factores de peso w1=0,05 y w2=0,95 con el fin de igualar las órdenes de magnitud de los índices IRPL e IMPT y darle importancia similar a ambos objetivos en el proceso de optimización. Sin embargo, el encargado del planeamiento del sistema puede seleccionar otros valores para darle más importancia a un objetivo en particular.

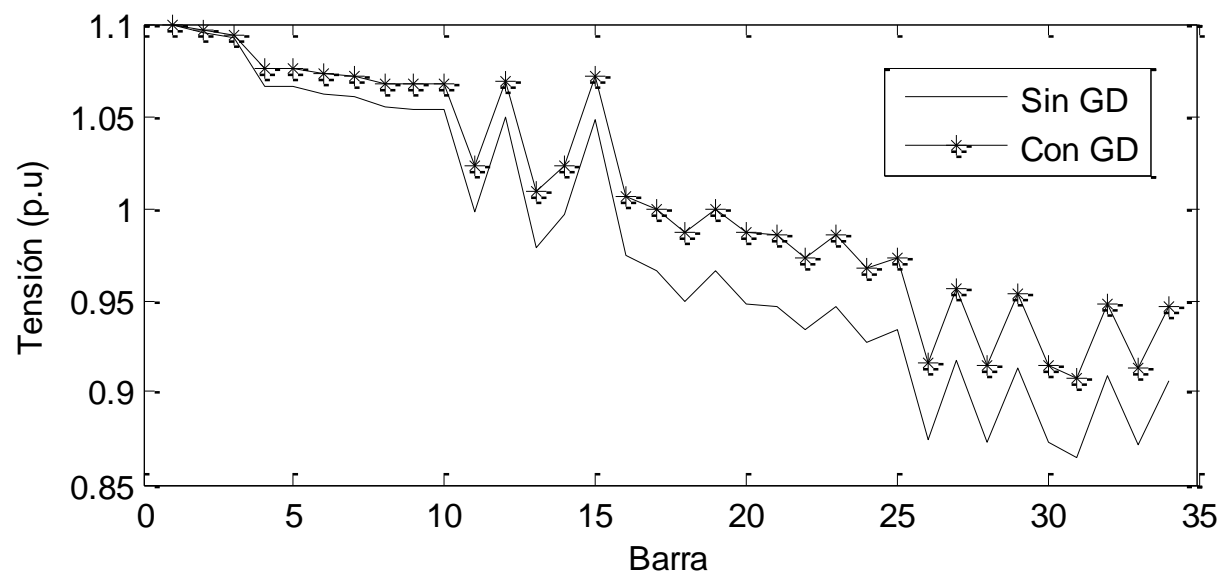

Fig.4: Perfil de tensión del sistema de 34 barras con y sin GD

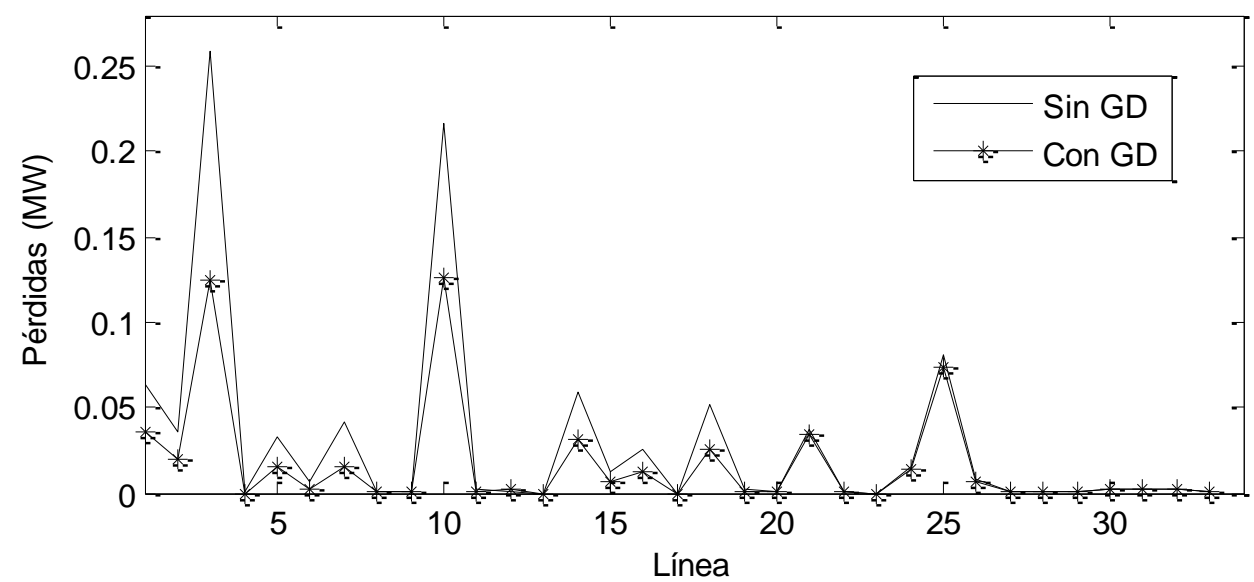

Fig.5: Pérdidas en las líneas del sistema de 34 barras con y sin GD

Tabla 3: Valores de IRPL e IMPT para candidatos de solución generados de forma aleatoria

\begin{tabular}{|c|c|c|c|}
\hline Simulación & IRPL & IMPT & IRPL/IMPT \\
\hline 1 & 41,884 & 1,513 & 27,682 \\
\hline 2 & 50,127 & 2,806 & 17,864 \\
\hline 3 & 43,297 & 2,124 & 20,385 \\
\hline 4 & 57,487 & 2,478 & 23,199 \\
\hline 5 & 34,329 & 1,878 & 18,280 \\
\hline 6 & 30,287 & 1,079 & 28,070 \\
\hline 7 & 44,238 & 2,567 & 17,233 \\
\hline 8 & 30,234 & 1,969 & 15,355 \\
\hline
\end{tabular}




\section{Resultados usando Recocido Simulado}

Para iniciar el RS se genera una solución base que cumpla con el criterio de máximo número de generadores. A partir de esta solución se exploran soluciones vecinas que serán aceptadas de acuerdo al criterio expresado en (19). Esto implica que al inicio del proceso se permitan empeoramientos de la función objetivo, pero a medida que evoluciona el proceso se restringe la probabilidad de aceptar soluciones de peor calidad. De esta forma, al principio de la búsqueda se privilegia la diversidad y al final de la misma se intensifica la búsqueda por mejores soluciones. Después de realizar varias corridas del algoritmo de RS se calibraron los parámetros de temperatura inicial y final en 3,0 y 0,5 , respectivamente, y en cada iteración se reduce la temperatura en 0,01 . En la Tabla 4 se muestra la mejor solución encontrada (después de 190 iteraciones) y en Fig. 6 se ilustra el proceso de convergencia del algoritmo de RS. En este caso el tiempo de cálculo fue 1,2 minutos.

Tabla 4: Mejor resultado usando RS

\begin{tabular}{|c|c|c|c|c|}
\hline Barra & Tamaño (MW) & \multirow{2}{*}{ W $_{1}$ IRPL } & \multirow{2}{*}{ w $_{2}$ IMPT } & FO \\
\hline 8 & 1,5 & & & \\
\cline { 1 - 2 } 2 & 1,75 & \multirow{2}{*}{2,9578} & \multirow{2}{*}{4,3747} & 7,3325 \\
\hline 22 & 2,0 & & & \\
\hline 29 & 1,5 & & & \\
\hline
\end{tabular}

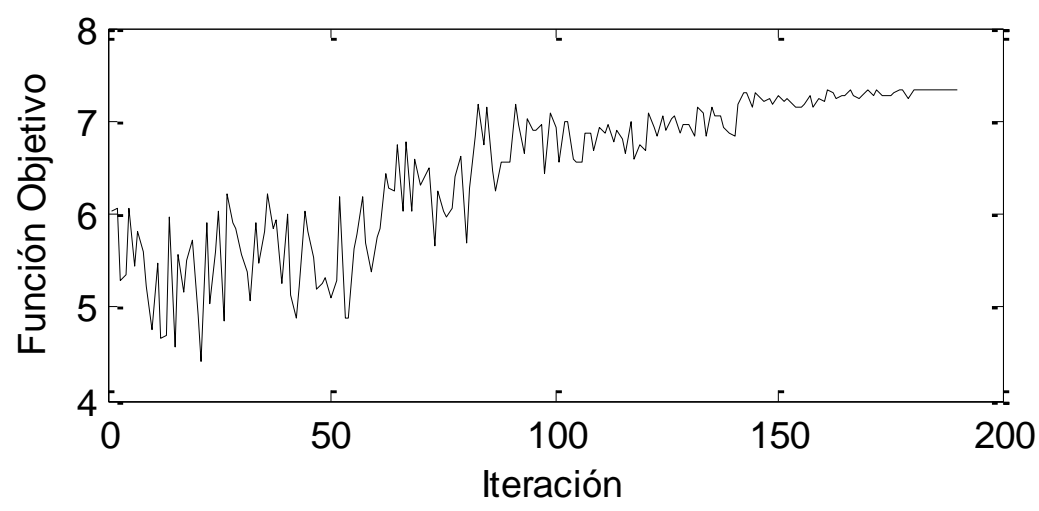

Fig. 6: Proceso de convergencia del algoritmo de RS

\section{Resultados usando BEVD}

Para realizar la BEVD se definieron dos estructuras de entorno: el tamaño y la ubicación de GD. Dada una solución base se exploran las estructuras de entorno buscando mejores individuos siguiendo las instrucciones ya descritas en la sección de Metodología. En la Fig. 7 se ilustran el proceso de búsqueda en las dos estructuras de entorno. La primera estructura de entorno es la posición, así que se selecciona la ubicación de una unidad de GD y se modifica en un vecindario dado para cada nueva posición se evalúan los índices IRPL e IMPT y se selecciona el mejor individuo. Posteriormente, sobre el mejor individuo del entorno se repite una búsqueda local pero esta vez modificando el tamaño de la GD. Para cada tamaño de GD se evalúan los índices y se determina el mejor individuo.

Entorno 1:

Cambio de posición

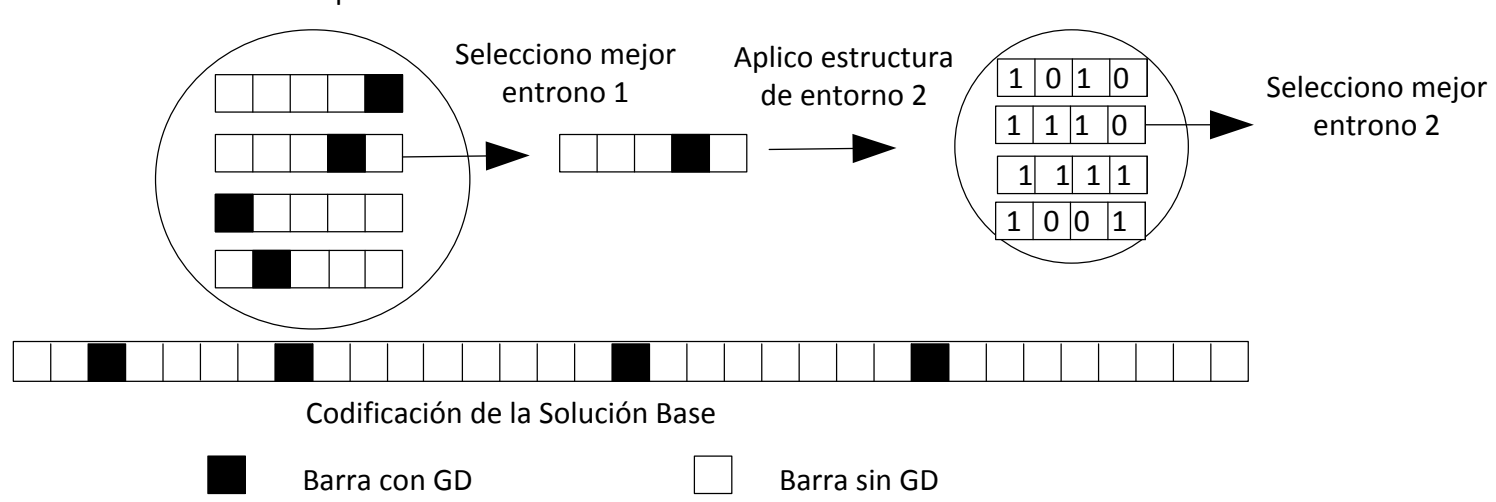

Entorno 2:

Cambio de tamaño

Fig.7: Proceso de búsqueda en las estructuras de entorno 
La búsqueda continúa hasta que pasa un número determinado de iteraciones sin mejora o hasta que se cumpla un número de iteraciones previamente establecido. En la Tabla 5 se ilustra el mejor resultado encontrado con la BEVD. En este caso se realizaron 22 iteraciones evaluando 8 individuos por estructura de entorno con un tiempo de cómputo de 2,1 minutos.

Tabla 5: Mejor resultado usando BEVD

\begin{tabular}{|c|c|c|c|c|}
\hline Barra & Tamaño $(\mathrm{MW})$ & \multirow{2}{*}{ W$_{1}$ IRPL } & W2IMPT & FO \\
\cline { 1 - 2 } 5 & 2,0 & & & \\
\cline { 1 - 2 } 30 & 2,0 & \multirow{3}{*}{3,9274} & \multirow{2}{*}{3,8313} & 7,7587 \\
\cline { 1 - 2 } 21 & 1,5 & & & \\
\hline 31 & 1,5 & & & \\
\hline
\end{tabular}

\section{Resultados usando el AG}

El AG implementado usa como codificación de solución la estructura de cromosoma ilustrada en la Tabla 2. Inicialmente se genera una población inicial de posibles soluciones a partir de las cuales se encuentran nuevas soluciones de mejor calidad mediante la implementación de los operadores de selección, recombinación y mutación descritos en la sección de Metodología. Para calibrar los parámetros del AG se realizaron varias pruebas modificando el tamaño de la población inicial y tasas de mutación y recombinación. Se observó que para poblaciones iniciales de pocos individuos se obtenían respuestas de baja calidad. Al aumentar el número de individuos en la población inicial la calidad de las soluciones mejora pero el tiempo de cómputo aumenta. La mejor solución se encontró con una población de 100 individuos y con tasas de mutación y recombinación del $10 \%$ cada una. El tiempo de cómputo para encontrar la mejor solución fue de 2,9 minutos después de 90 iteraciones. En la Tabla 6 se ilustra el mejor resultado obtenido. En Fig. 8 se ilustra el proceso de convergencia del AG para diferentes pruebas. Se puede observar que a pesar de que las poblaciones iniciales son de diferente calidad, el AG encuentra soluciones de calidad similar.

Tabla 6: Mejor resultado usando AG

\begin{tabular}{|c|c|c|c|c|}
\hline Barra & Tamaño (MW) & $\mathrm{W}_{1} \mathrm{IRPL}$ & $\mathrm{w}_{2}$ IMPT & $\mathrm{FO}$ \\
\hline 10 & 2,0 & \multirow{4}{*}{3,8887} & \multirow{4}{*}{3,7836} & \multirow{4}{*}{7,6723} \\
\hline 15 & 1,5 & & & \\
\hline 26 & 1,5 & & & \\
\hline 29 & 1,25 & & & \\
\hline
\end{tabular}

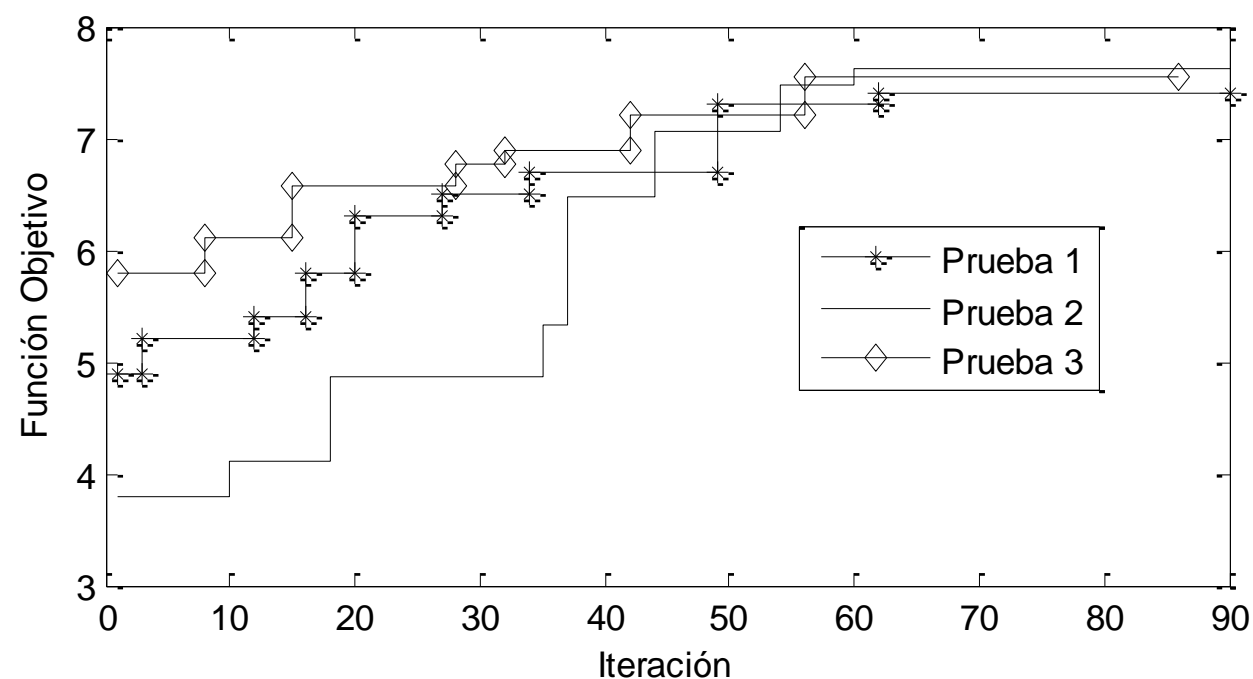

Fig.8: Proceso de convergencia del AG para diferentes pruebas 


\section{Resultados usando el $\mathrm{AGH}$}

Como se mencionó anteriormente el AGH implementado combina el AG descrito en la sección anterior con la BEVD. En este caso, después de aplicar los operadores tradicionales del AG y antes de realizar la sustitución en la población se realiza una BEVD para aumentar la calidad de los individuos en cada iteración. Para el AGH se utilizaron los mismos parámetros que se calibraron con el AG tradicional. En la Tabla 7 se presenta la mejor solución encontrada. Se pudo observar para todas las pruebas que el tiempo de cómputo es considerablemente mayor que el requerido por los otros algoritmos implementados; sin embargo, la calidad de las respuestas es mejor. La mejor respuesta se encontró después de solo 6 iteraciones con un tiempo de cómputo de 12,6 minutos.

Tabla 7: Mejor resultado usando AGH

\begin{tabular}{|c|c|c|c|c|}
\hline Barra & Tamaño (MW) & \multirow{2}{*}{ w $_{1}$ IRPL } & w2IMPT & FO \\
\hline 12 & 1,75 & & & \\
\cline { 1 - 2 } 13 & 2,0 & \multirow{2}{*}{4,0573} & \multirow{2}{*}{3,9201} & 7,9774 \\
\cline { 1 - 2 } 22 & 1,0 & & & \\
\hline 30 & 1,25 & & & \\
\hline
\end{tabular}

\section{Comparación}

Se pudo observar que el mejor resultado fue obtenido con el AGH; sin embargo, este es el método que más tiempo toma en encontrar una solución de alta calidad. Por otro lado, el algoritmo en converger más rápido fue el Recocido Simulado. A pesar de que esta metodología es mucho más rápida, las soluciones obtenidas con las otras metodologías fueron de mejor calidad. La BEVD requiere menos iteraciones que el RS para converger, sin embargo el tiempo de cálculo por iteración es mayor, ya que se deben evaluar, por iteración, dos estructuras de vecindad. En cuanto al AG y al AGH se pudo comprobar que se para encontrar soluciones de alta calidad es necesario usar poblaciones iniciales grandes (100 individuos). En Fig. 9 se compara el tiempo de cómputo para cada uno de los métodos utilizados, donde se destaca el AGH. En Fig. 10 se ilustra la calidad de las soluciones obtenidas con cada uno de los algoritmos. En general se puede observar que las soluciones son de calidad similar. Para la mejor solución encontrada (ver Tabla 7) se pudo verificar una reducción de pérdidas del $80,2 \%$ con respecto al caso base sin GD.

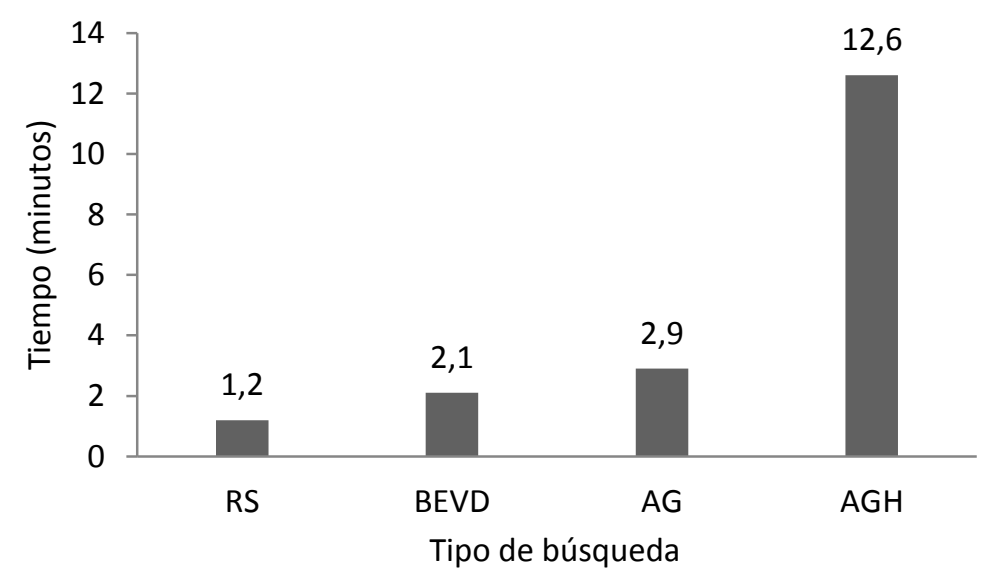

Fig.9: Comparación de tiempos de búsqueda de los algoritmos bajo estudio

\section{CONCLUSIONES}

En este artículo se comparó el desempeño de diferentes algoritmos para la ubicación y dimensionamiento de GD en sistemas de distribución. Cuatro algoritmos fueron considerados: 1) RS, 2) BEVD, 3) AG y 4) AGH. Todas las metodologías implementadas tuvieron éxito en encontrar soluciones de alta calidad, con diferentes tiempos de cómputo. En las pruebas realizadas se obtuvieron mejores resultados con el AGH, que combina los métodos 2 y 3 . En todos los resultados se pudo observar que el porcentaje óptimo de penetración de GD, para el sistema de prueba varía entre 6 y $7 \mathrm{MW}$ instalados en las barras iniciales y finales, en unidades de GD de 1 a 2 MW. Adicionalmente, la correcta ubicación y dimensionamiento de la GD permitió mejorar sustancialmente el perfil de tensión de la red y reducir las pérdidas en un $80,2 \%$ para el sistema de prueba. En lo referente a tiempos de cómputo, el método más rápido fue el de RS, por otro lado, la BEVD y el AGH, a pesar de requerir más tiempo de cómputo, mostraron mejores resultados. 


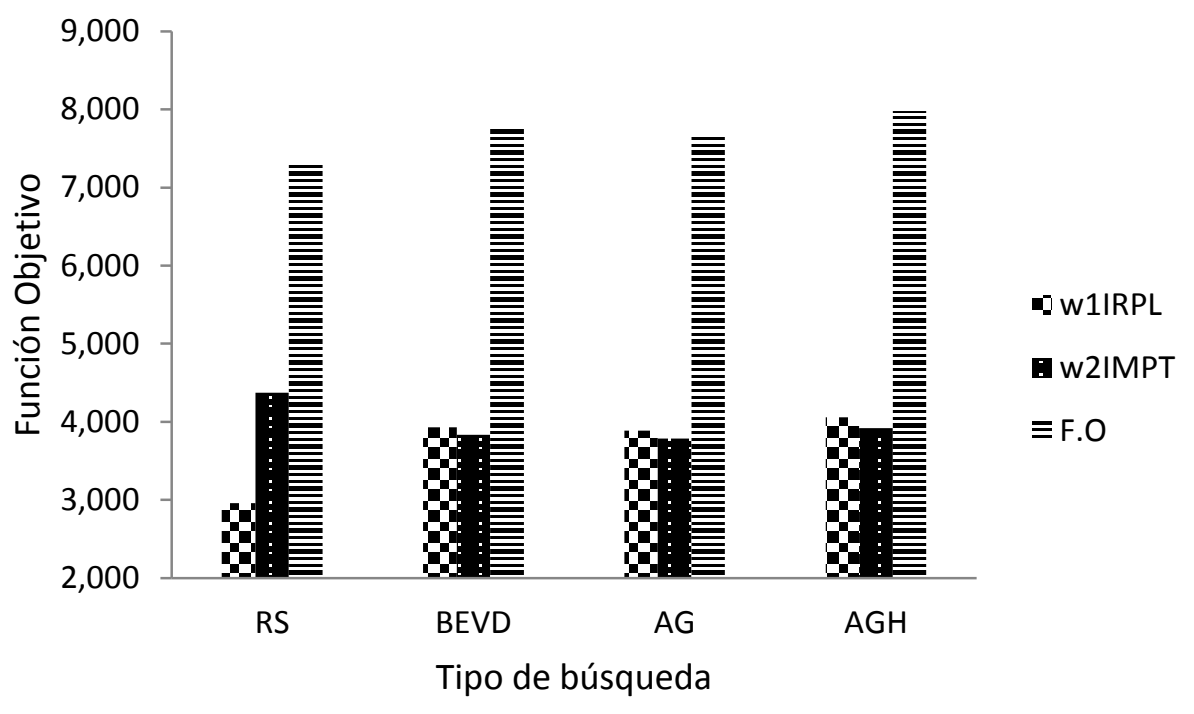

Fig.10: Comparación de soluciones obtenidas con los algoritmos propuestos

\section{AGRADECIMIENTOS}

Los autores agradecen al, Programa de Sostenibilidad 2014-2015 de la Universidad de Antioquia por el apoyo económico.

\section{REFERENCIAS}

Akorede, M.F., Hizam, H., Aris, I., y Kadir, M.Z.A., Effective method for optimal allocation of distributed generation units in meshed electric power systems, IET Generation Transmission \& Distribution., 5(2), 276287, (2011)

Aly, A.I., Hegazy. Y.G. and Alsharkwy, M.A., A simulated annealing algorithm for multi-objective distribured generation planning, IEEE Power and Energy Society General Meeting, Minneapolis, USA July. (2010)

Anaut, D., Di Mauro, G., Meschino, G., y Suarez, J., Optimización de Redes Eléctricas Mediante la Aplicación de Algoritmos Genéticos, Revista Inf. Tecnol. Cit: 20(4), 137-148, (2009)

Chen, C. and Duan S., Optimal allocation of distributed generation and energy storage system in microgrids., IET Generation Transmission \& Distribution., 8(6), 581-589, (2014)

Chiradeja, P.; Ramakumar, R.; An approach to quantify the technical benefits of distributed generation, IEEE Trans. Power Syst., 19 (4), 764-773, (2004)

Gandomkar, M. Vakilian, M. and Ehsan M., A combination of genetic algorithm and simulated annealing for optimal DG allocation in distribution networks., Canadian Conference on Electrical and Computer Engineering pp 645-648, (2005)

Georgilakis, P.S. and Hatziargyriou N.D., Optimal distributed generation placement in power distribution networks: models, methods and future research, IEEE Transactions on Power Systems, 28(3), 3420-3428, (2013)

Glover J., G.A., Handbook of Metaheuristics, New York. Kluwer Academic Plublishers, (2003)

Hansen, P., Mladenovic, N. and J.A. Moreno Pérez. Búsqueda de entorno Variable, Revista Iberoamericana de inteligencia Artificial. 19 (1), 77-92, (2003)

Hung, D.Q. and Mithualananthan, N., Multiple distributed generation placement in primary distribution networks for loss reduction, IEEE Transactions on Power Systems, 60(4), 1700-1707, (2013) 
Hung, D.Q., Mithualananthan, N., and Bansal R.C., Analytical expressions for DG allocation in primary distribution networks, IEEE Transactions on Power Systems, 25(3), 814-820, (2010)

Jonghe De C., Hobbs, B.F. and Belmans, R. Optimal generation mix with short-term demand response and wind penetration, IEEE Transactions on Power Systems, 27(2),830-839, (2011)

Kirkpatrick, S. Gellat, C.D. and M.P. Vecci. Optimization by simulated annealing" Journal of Statistical Physics, 34(5), 975-986, (1983)

Leopold S. and J.M Monzon, Identificación y ajuste paramétrico de una máquina trifásica de inducción magnética usando algoritmos genéticos Revista Inf. Tecnol. Cit: 21(3) 105-114 (2010)

Lopez-Lezama J.M., Padilha-Feltrin A., Contreras A. and Muñoz J.I., Optimal contract pricing of distributed generation in distribution systems, IEEE Transactions on Power Systems, 26(1), 128-136, (2011)

Maciel, R.S., and Padilha-Feltrin, A., Distributed generation impact evaluation using a multi-objective Tabu Search, $15^{\text {th }}$ International Conference on Intelligent System Application to Power System (ISAP), Curitiba, Brazil, July. (2009)

Soroudi, A., Ehsan, M., Caire, R., and Hadjsaid, N., Hybrid immune-genetic algorithm method for benefit maximization of distribution network operatros and distributed generation owners in a deregulated environment., IET Generation Transmission \& Distribution., 5(9), 961-972, (2011)

Soroudi, A., y Afrasiab M., Binary PSO-based dynamic multi-objective model for distributed generation planning under uncertainty., IET Generation Transmission \& Distribution., 6(2), 67-78, (2012)

Tan, W.S., Hassan, M.Y., Majid, M.S., Rahman, H.A., Allocation and sizing of DG using Cuckoo search Algorithm, IEEE International Conference on Power and Energy, Kota Kinabalu, Malasya, Dec. (2012)

Tan, W.S., Hassan, M.Y., Rahman, H.A., and Abdullah, M.P., Multi-distributed generation planning using hybrid particle swarm optimization-gravitational search algorithm., IET Generation Transmission \& Distribution., 7(9), 929-942, (2013)

Zhao, Y., An, Y., and Ai, Q., Research on size and location of distributed generation with vulnerable node identification in the active distribution network., IET Generation Transmission \& Distribution., 8(11), 18011809, (2014)

Zimmerman, R.D., Murillo-Sánchez, C.E., and Thomas R.J., Matpower: a steady-state operations planning and analysis tools for power systems research and education., IEEE Transactions on Power Systems., 26(1),12-19, (2011) 
